\title{
Sarcoma de Ewing pélvico: O grande imitador*
}

\section{Pelvic Ewing Sarcoma: the Great Mimicker}

\author{
Francisco Lima-Bernardes ${ }^{1}$ Diogo Manuel Soares $^{1(0)}$ Joana Monteiro Pereira ${ }^{1(0)}$ Isabel Catarino ${ }^{10}$

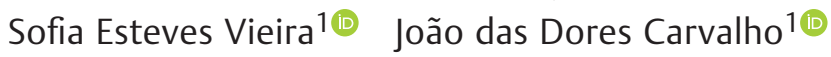 \\ ${ }^{1}$ Departamento de Ortopedia e Traumatologia, Centro Hospitalar do \\ Tâmega e Sousa, Penafiel, Portugal \\ Endereço para correspondência Francisco Manuel Flores Lima- \\ Bernardes, MD, Departamento de Ortopedia e Traumatologia, Centro \\ Hospitalar do Tâmega e Sousa, Penafiel, Portugal \\ Rev Bras Ortop \\ (e-mail: franciscolimabernardes@gmail.com).
}

\section{Resumo \\ Palavras-chave \\ - sarcoma de Ewing \\ - neoplasias pélvicas \\ - granuloma eosinofílico \\ - osteomielite \\ - anti-inflamatórios não esteroides}

O sarcoma de Ewing é o tumor ósseo maligno da pelve mais comum em crianças e adultos jovens. Mesmo com tratamento agressivo, sua taxa de sobrevivência está entre as piores. A apresentação clássica pode não ser a regra. Ele pode simular clinicamente, imaginologicamente e histopatologicamente outras entidades não malignas. Portanto, sua suspeita não deve ser negligenciada. Relatamos dois casos de sarcoma pélvico: o primeiro imitando granuloma eosinofílico e o segundo imitando osteomielite. Neste último, também relatamos um achado atípico de sua história natural: uma resposta inicial ao antibiótico e ao tratamento anti-inflamatório. Em ambos os casos, destacamos a possibilidade de uma biópsia óssea percutânea inconclusiva e a importância da imunoquímica e da citogenética para o diagnóstico definitivo.

Ewing sarcoma is the most common malignant bone tumor of the pelvis in children and young adults. Even with aggressive treatment, its survival rate is amongst the poorest. Classical presentation may not be the rule. It may simulate clinically, imagiologically and histopathologically other nonmalignant entities. Therefore, its suspicion should not be overlooked. We report two cases of pelvic Ewing sarcoma: the first mimicking eosinophilic granuloma, and the second mimicking osteomyelitis. In the latter, we also report an atypical finding of its natural history: an initial response to antibiotic and antiinflammatory treatment. In both cases, we highlight the possibility of an inconclusive percutaneous bone biopsy and the importance of immunochemistry and cytogenetics for the definitive diagnosis.

\section{Introdução}

O sarcoma de Ewing (ES, na sigla em inglês) é um tumor ósseo primário. Em > 50\% dos casos, um componente de tecido mole

Trabalho desenvolvido no Departamento de Ortopedia e Traumatologia, Centro Hospitalar do Tâmega e Sousa, Penafiel, Portugal.

recebido

18 de Julho de 2020

aceito

17 de Setembro de 2020
DOI https://doi.org/

10.1055/s-0040-1722582.

ISSN 0102-3616. está associado. ${ }^{1} \mathrm{O}$ ES é raro, com incidência anual de 2,93 por milhão de indivíduos. ${ }^{2,3}$ Entre os tumores ósseos, ele é um dos mais letais, com alta propensão para recidiva e metástase distante (predominantemente pulmonar). Geralmente, ele afeta a diáfise e a metáfise de ossos longos. ${ }^{4,5}$ É raro em pacientes com mais de 30 anos, com 90\% dos afetados com (c) 2021. Sociedade Brasileira de Ortopedia e Traumatologia. All rights reserved.

This is an open access article published by Thieme under the terms of the Creative Commons Attribution-NonDerivative-NonCommercial-License, permitting copying and reproduction so long as the original work is given appropriate credit. Contents may not be used for commercial purposes, or adapted, remixed, transformed or built upon. (https://creativecommons.org/ licenses/by-nc-nd/4.0/)

Thieme Revinter Publicações Ltda., Rua do Matoso 170, Rio de Janeiro, RJ, CEP 20270-135, Brazil 


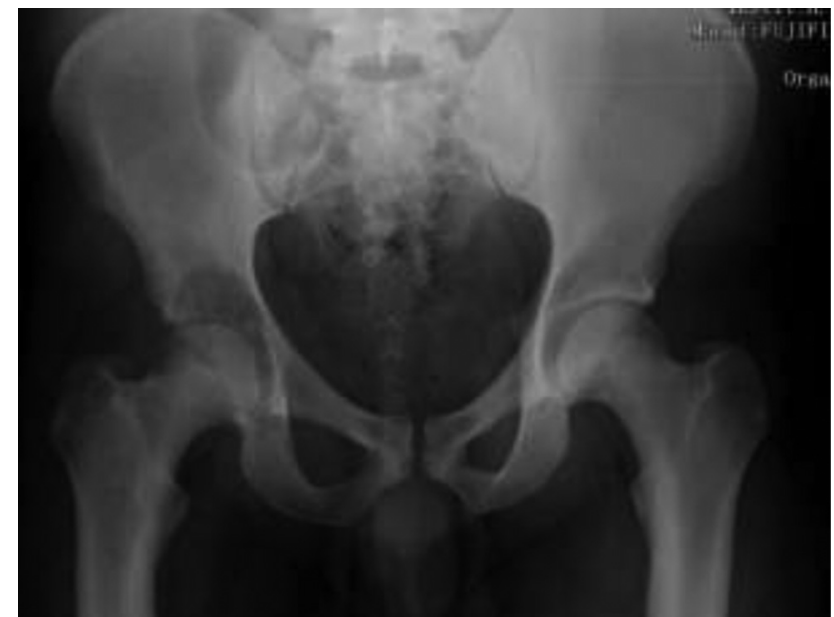

Fig. 1 Radiografia na admissão mostrando uma lesão radiolúcida do acetábulo direito.

idade $<20$ anos. ${ }^{4}$ Nesta faixa etária, ele é o tumor pélvico mais comum. O prognóstico do ES pélvico é ruim, com taxa de sobrevivência de 5 anos para $50 \%$ dos pacientes. ${ }^{6}$ Os sintomas mais comuns são dor noturna (90\%) e inchaço (70\%), este último de difícil avaliação no ES pélvico. Febre, perda de peso, anemia e aumento de marcadores inflamatórios são manifestações inespecíficas incomuns. ${ }^{1,7,8}$ Radiografias apresentam destruição lítica e formação óssea subperiosteal semelhante a "esfolar cebola". 1,4,5,8 Essas características são mais facilmente detectadas em ossos longos em comparação com a pelve. O valor das radiografias na pélvis é comprometido pela anatomia do osso ilílico e de estruturas de estruturas sobrejacentes. ${ }^{1,8}$ A tomografia computadorizada (TC) permite uma melhor avaliação da destruição óssea e pode mostrar um componente de tecido mole. ${ }^{1,5}$ A ressonância magnética (RM) precoce para caracterização de tecidos moles e, em menor grau, a identificação de uma "zona de transição acentuada", é útil. ${ }^{3,7}$ Esses achados clínicos e imaginológicos não específicos podem estar presentes em condições como carcinoma metastático, linfoma maligno, osteomielite, ${ }^{4}$ displasia fibrosa $^{5}$ ou granuloma eosinofílico (EG, na sigla em inglês), ${ }^{9}$ tornando seu diagnóstico precoce desafiador. ${ }^{1-9}$ O diagnóstico definitivo é feito por biópsia aberta e exame histológico combinado com imunoquímica e citogenética. ${ }^{1,4}$ A falta ou insuficiência de espécimes histológicos por biópsia percutânea pode levar ao seu diagnóstico errado. ${ }^{2,4}$ Relatamos dois casos de ES que frequentaram nosso departamento nos últimos 3 anos, o primeiro imitando EG e o segundo osteomielite. Nosso objetivo é destacar armadilhas sobre o diagnóstico de ES pélvico e relatar um achado atípico de sua história natural: uma resposta inicial ao antibiótico e ao tratamento antiinflamatório, descrito na literatura apenas uma vez. ${ }^{4}$

\section{Relatório de Caso 1}

Um homem de 20 anos se apresentou ao nosso pronto-socorro com histórico de 6 meses de dor mecânica e inflamatória no quadril direito e febre recorrente. Os exames de sangue estavam normais. As radiografias revelaram uma lesão radiolúcida que afetava o acetábulo direito (-Fig. 1). A TC mostrou uma lesão osteolítica envolvendo a borda acetabular posterior, com ruptura cortical (-Fig. 2). A RM pélvica mostrou um componente de tecido mole associado ( - Fig. 3). Esta lesão foi consistente com ES, não excluindo EG. O exame histopatológico após a biópsia óssea percutânea guiada pela imagem foi consistente com EG. Devido ao risco de fratura, o paciente foi submetido a curetagem e colocação de autoenxerto. O exame histopatológico de novas amostras foi consistente com ES, posteriormente confirmada por imunoquímica e citogenética. O paciente foi transferido para um centro especializado.

\section{Relatório de Caso 2}

Um homem de 30 anos, com histórico anterior de sífilis e vício em drogas, se apresentou ao nosso departamento de emergência com 1 mês de dor inguinal direita sem sintomas constitucionais. Os exames de sangue demonstraram uma
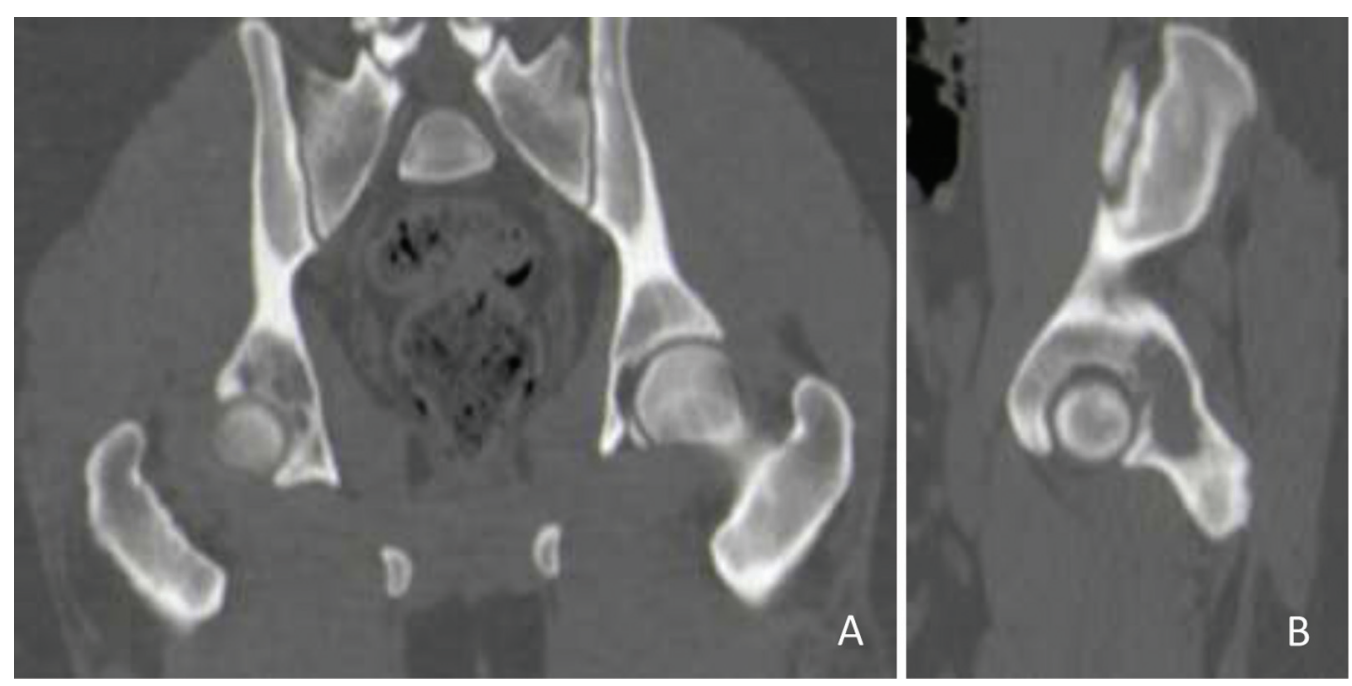

Fig. 2 Tomografia computadorizada na admissão mostrando uma lesão osteolítica da borda acetabular posterior direita com ruptura cortical. Vistas coronal (A) e sagital (B). 

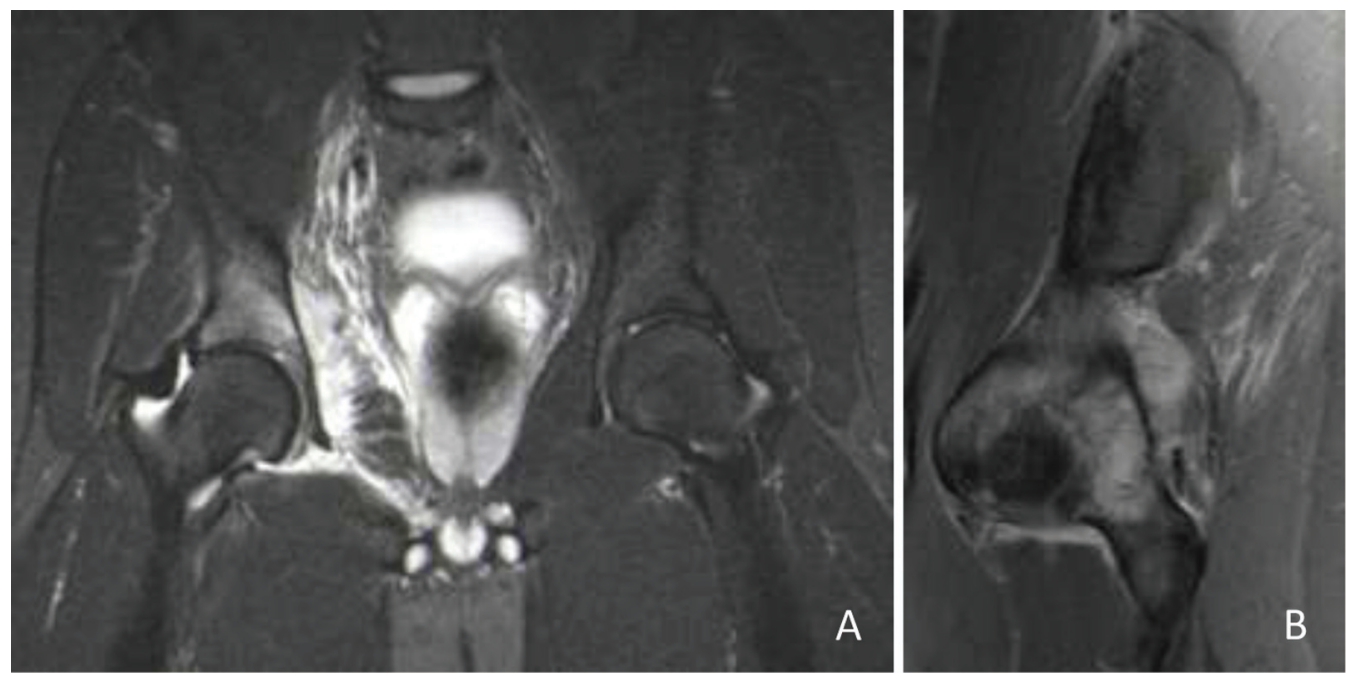

Fig. 3 Ressonância magnética mostrando uma massa de tecido mole associada. Vistas coronal (A) e sagital (B).

elevada contagem de células brancas $\left(15,9 \times 10^{9}\right.$ células/L) e aumento da proteína C-reativa $(104 \mathrm{mg} / \mathrm{L})$. A hemoglobina e a taxa de sedimentação de eritrócitos estavam normais. As radiografias foram inconclusivas ( $\mathbf{- F i g . ~ 4 A )}$ ). Os achados de TC e de RM foram consistentes com uma lesão inflamatória envolvendo o ramo isquiopúbico direito e os músculos obturadores, com abscesso associado, tornando o diagnóstico de osteomielite mais provável ( - Figs. 4B e 5A). Devido ao aparecimento da febre e ao aumento dos marcadores inflamatórios, foi iniciada a terapia com antibióticos empíricos (ceftriaxona intravenosa, $2 \mathrm{~g} / \mathrm{dia}$ ). Antes da terapia antibiótica, foram coletadas culturas sanguíneas e foi realizada uma drenagem percutânea, mas nenhum agente foi isolado. 0 paciente respondeu bem ao antibiótico e ao tratamento antiinflamatório com resolução da febre e da dor. Após 15 dias de tratamento, houve uma diminuição considerável na contagem de células brancas $\left(11,5 \times 10^{9}\right.$ células/L) e proteína C-reativa $(18,0 \mathrm{mg} / \mathrm{L})$. No entanto, a RM mostrou persistência da lesão (- Fig. 5B). O paciente foi submetido a uma biópsia excisional. $\mathrm{O}$ exame histopatológico foi consistente com ES, mais tarde confirmado por imunoquímica e citogenética. O paciente foi transferido para um centro especializado.

\section{Discussão}

O ES pode levantar suspeita de malignidade em adultos jovens quando envolve ossos longos em um local típico com dor associada e massa de tecido mole palpável. ${ }^{5} \mathrm{Na}$ pelve, este último é difícil e, portanto, os sintomas constitucionais não devem ser negligenciados. Por outro lado, o EG, uma condição benigna semelhante ao tumor, geralmente afeta ossos planos. Radiologicamente, a fase aguda do EG mostra a osteólise com margens mal definidas semelhantes às do ES. Na RM, o envolvimento da medula óssea e um componente de tecido mole associado também podem estar presentes. Como as características imaginológicas são semelhantes, a biópsia da lesão é indispensável para o diagnóstico correto. ${ }^{9}$ No caso 1 , as amostras coletadas por biópsia percutânea não foram representativas, levando ao diagnóstico errado do EG. Portanto, quando as características clínicas e imaginológicas são

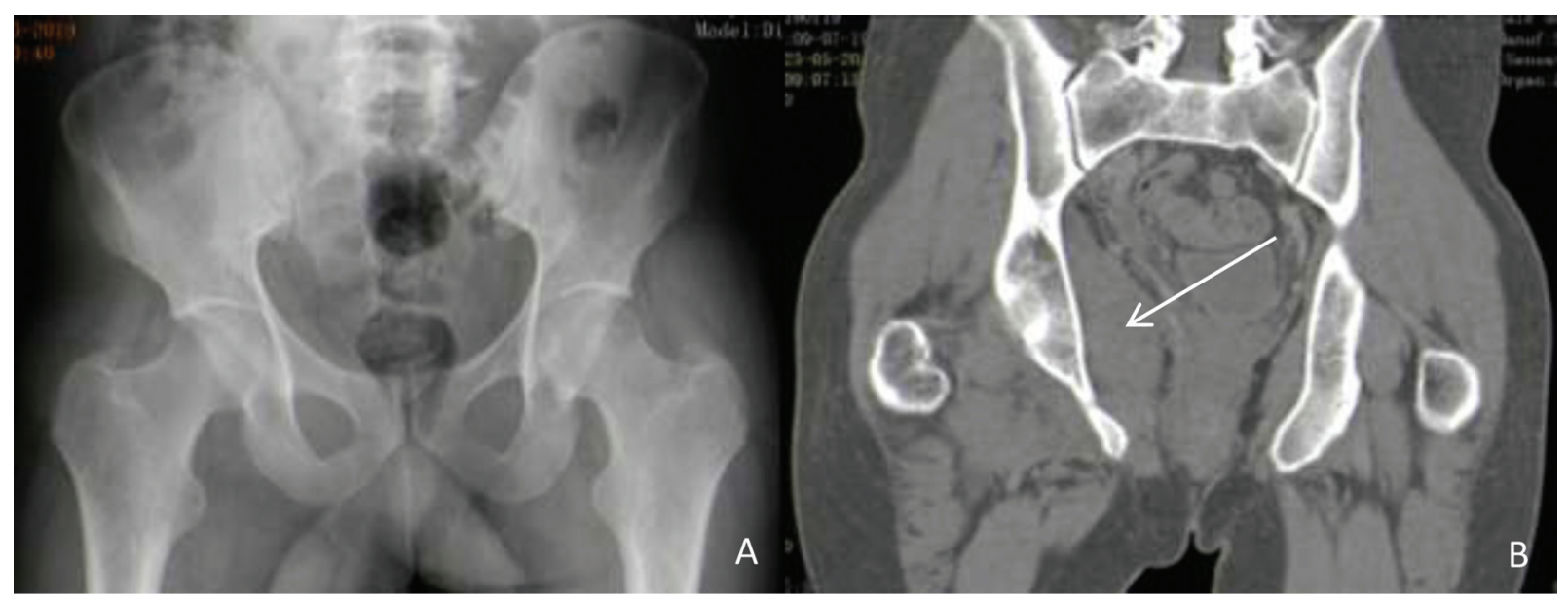

Fig. 4 Radiografia inconclusiva na admissão (A) e tomografia computadorizada mostrando alargamento dos músculos obturadores direito (B). 


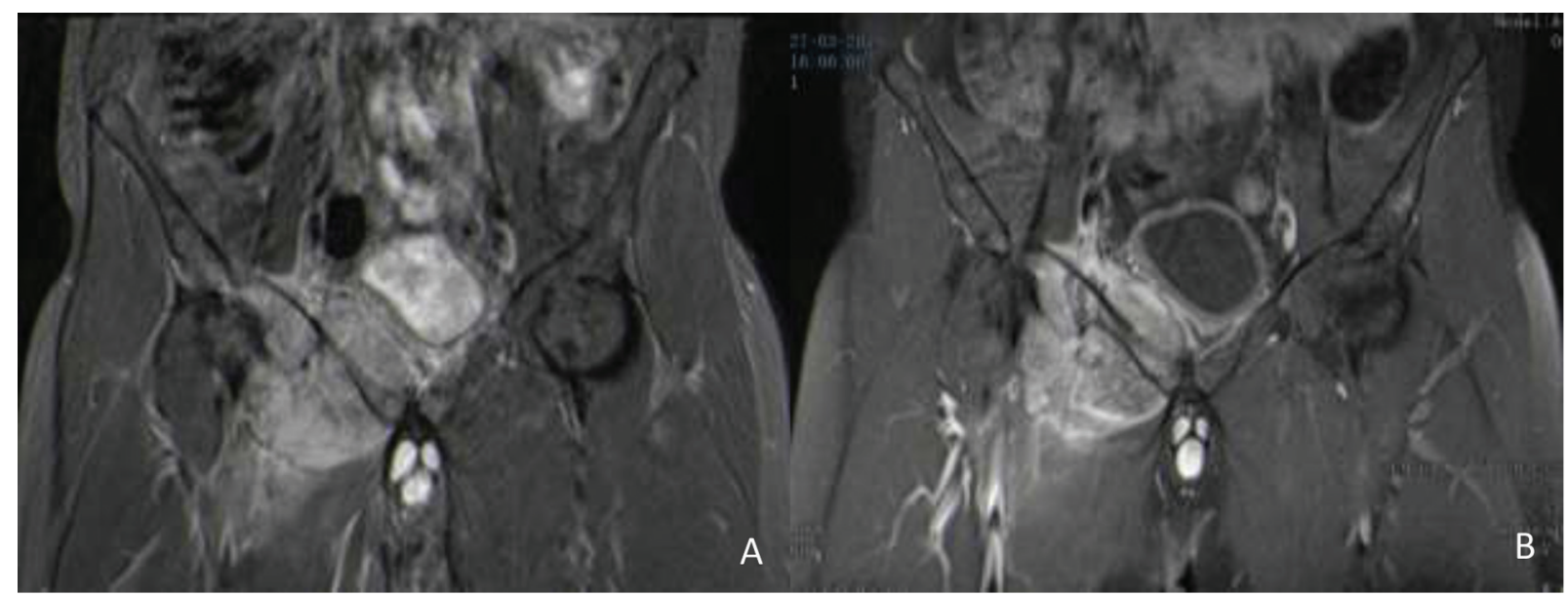

Fig. 5 Ressonância magnética inicial mostrando uma lesão envolvendo o ramo isquiopúbico direito e os músculos obturadores (A) e ressonância magnética após tratamento antibiótico mostrando persistência da lesão (B).

consistentes com o ES, uma biópsia percutânea negativa não deve descartá-la, e uma biópsia excisional deve ser realizada. Para minimizar erros de diagnóstico e contaminação de vias biópsias, biópsias devem ser realizadas por cirurgiões com experiência em tumores ósseos. ${ }^{10}$

No caso 2, a apresentação foi atípica. A idade, histórico médico anterior, achados imaginológicos e bioquímicos foram consistentes com osteomielite. A esterilidade da biópsia e das culturas sanguíneas não excluiu seu diagnóstico, uma vez que até $26 \%$ dos casos de osteomielite são diagnosticados sem confirmação cultural e apoiados retrospectivamente por sua resolução após a terapia com antibióticos. ${ }^{4}$ Além disso, nosso paciente respondeu ao antibiótico e ao tratamento anti-inflamatório. Esta resposta pode ser explicada pela associação de níveis elevados de metabólitos ácidos aracidônicos e de prostaglandinas às malignidades. A inibição da via do ácido aracidônico por antibiótico e agentes anti-inflamatórios não esteroides pode ter modulado os sintomas clínicos e o crescimento do tumor. ${ }^{4}$

\section{Conclusões}

O diagnóstico de ES não deve ser negligenciado na avaliação das lesões pélvicas, e o diagnóstico por exclusão deve ser a regra. Se as características clínicas e imaginológicas condizem com seu diagnóstico, uma biópsia percutânea negativa não deve ser descartada, e uma biópsia excisional deve ser realizada. Se as características clínicas e imaginológicas são consistentes com osteomielite, uma resposta inicial ao antibiótico e à terapia anti-inflamatória não permite sua exclusão, mas recomenda-se a observação atenta.
Conflito de Interesses

Os autores não têm conflitos de interesse a declarar.

\section{Referências}

1 Halwai MA, Mir BA, Wani MM, Bashir A, Hussain A. Ewing's sarcoma of the ilium mimicking inflammatory arthritis of the hip: a case report. Cases J 2009;2:6487

2 McCarville MB, Chen JY, Coleman JL, et al. Distinguishing osteomyelitis from Ewing sarcoma on radiography and MRI. AJR Am J Roentgenol 2015;205(03):640-650

3 Kasalak Ö, Overbosch J, Adams HJ, et al. Diagnostic value of MRI signs in differentiating Ewing sarcoma from osteomyelitis. Acta Radiol 2019;60(02):204-212

4 Tow BP, Tan MH. Delayed diagnosis of Ewing's sarcoma of the right humerus initially treated as chronic osteomyelitis: a case report. J Orthop Surg (Hong Kong) 2005;13(01):88-92

5 Flores M, Caram A, Derrick E, Reith JD, Bancroft L, Scherer K. Ewing sarcoma of the pelvis with an atypical radiographic appearance: a mimicker of non-malignant etiologies. Cureus 2016;8(09):e787

6 Natarajan MV, Sameer MM, Bose JC, Dheep K. Surgical management of pelvic Ewing's sarcoma. Indian J Orthop 2010;44(04): 397-401

7 Ray P, Girach J, Sanghrajka AP. Ewing's sarcoma of the pelvis: an unusual, but not to be missed, cause of an irritable hip. BMJ Case Rep 2016;2016:bcr2015213782

8 Alshaya OS, Abbasher MI, Wani MM. Ewing's sarcoma of ilium presenting as right lower quadrant pain. Case Rep Orthop 2015; 2015:629601

9 Ando A, Hatori M, Hosaka M, Hagiwara Y, Kita A, Itoi E. Eosinophilic granuloma arising from the pelvis in children: A report of three cases. Ups J Med Sci 2008;113(02):209-216

10 Garcia JG, Marques DS, Viola DCM, Petrilli MT, Alves MTS, JesusGarcia Filho R. Biopsy Path Contamination in Primary Bone Sarcomas. Rev Bras Ortop (Sao Paulo) 2019;54(01):33-36 